\title{
Cancer Risk Assessment and Screening; A Challenge for Clinical Pathology Service?
}

\author{
Siti Boedina Kresno \\ Department of Clinical Pathology, Dharmais National Cancer Center Hospital, Jakarta, Indonesia. E-mail: sbkresno@gmail.com
}

\begin{abstract}
There is evidence demonstrating that cancer etiology is multi-factorial and modification of risk factors has achieved cancer prevention. There is therefore a need to advance the understanding of cancer etiology through interaction effects between risk factors when estimating the contribution of an individual to the cancer burden in a population. It has been known that cancer may arise from genetic susceptibility to the disease as an intrinsic factor; however, non-intrinsic factors drive most cancer risk as well and highlight the need for cancer prevention. Are our clinical pathologists aware of these facts?. Are they ready to understand and to provide an excellent test with good expertise?. Hereditary cancer testing is typically performed using gene panels, which may be either cancer-specific or pan-cancer to assess risk for a defined or broader range of cancers, respectively. Given the clinical implications of hereditary cancer testing, diagnostic laboratories must develop high-quality panel tests, which serve a broad, genetically diverse patient population. The result will determine a patient's eligibility for targeted therapy, for instance, or lead a patient to prophylactic surgery, chemoprevention, and surveillance. This review will introduce the definitions of intrinsic and non-intrinsic risk factors, which have been employed in recent work and how evidence for their effects on the cancer burden in human subjects has been obtained. Genetic testing of cancer susceptibility genes by use of liquid biopsies and New Generation Sequencing (NGS) is now widely applied in clinical practice to predict the risk of developing cancer, help diagnosis, and treatment monitoring.
\end{abstract}

Keywords: Cancer risk factors, intrinsic, non-intrinsic, gene variants, multi-panel gene testing

\section{INTRODUCTION}

Cancer shows a very complex etiopathogenesis, and in the course of its evolution, several factors interact one with the other, e.g. interaction between one gene with the other or with environmental factors (gene-gene or gene-environment interplay). ${ }^{1}$ There has been significant progress in our understanding of cancer etiology as well as achievements in prevention, early detection, and treatment, which have led to declining cancer mortality in the past few decades. Despite these advances, certain cancers continue to increase in different parts of the world related to longer lifespans and changing patterns of cancer risk factors. ${ }^{1,2}$ Cancer risk assessment and screening through the application of liquid biopsy plays an important role in declining cancer mortality., Laboratory results will determine the patient's eligibility for targeted therapy or lead the patient to have change(s) of lifestyle, prophylactic surgery, chemoprevention, or surveillance. It is a challenge for clinical pathologists.

\section{CANCER RISK FACTORS}

Certain individuals generate cancer at some point in their life, while others do not and certain human tissue is susceptible to cancer more than others. ${ }^{2}$ These facts lead to the debate on the nature and contribution of cancer risks and the importance of extrinsic versus intrinsic risk factors. Nowadays, it is clear that extrinsic and intrinsic risk factors are not always exclusive, indicating a strong interdependence among those factors. ${ }^{2}$

In families whose members are affected with cancer at their young age, hereditary factors might be one of the etiology of cancer, such as Li Fraumeni syndrome for breast cancer and Cowden syndrome for breast, thyroid, and endometrial tumor or Lynch syndrome for colorectal cancer. ${ }^{7,8}$ Many cancers show family clustering or family aggregation. This might be due to the interplay between genes together with the same environmental factors; however, most cancers with family clustering are linked to a strong predisposition due to germline mutation passed on to the family members. In 
addition to genetic factors, epigenetic factors may be a more important risk factor of cancer because most cancers are sporadic or non-hereditary. ${ }^{7,9}$

The key-points of cancer predisposition are as follows: ${ }^{7}$ Most cancers have a multifactorial etiology and are attributable to a varying blend of genetic and environmental factors; only about $5 \%$ of common cancers are due to a strongly inherited susceptibility; A strong family history of the same or related cancers on the same side of the family (especially early-onset and multiple cancers) suggests a significant genetic predisposition and an increased risk of cancer to individuals in that family; A minority of cancers are due to monogenic cancer predisposition syndromes in which there is Mendelian inheritance with incomplete penetrance, conferring increased susceptibility to a characteristic spectrum of cancers; Identification of people with increased risk of cancer by considering the family genealogy is crucial as surveillance and prophylactic surgery may be indicated. Targeted chemoprophylaxis is under investigation although some has been encouraged ${ }^{5,6}$ Cancer genetics services and family clinics play an important role to evaluate the risk of an inherited cancer predisposition by performing genetic testing, surveillance, and preventive actions

\section{CLASSIFICATION OF CANCER RISK FACTORS}

According to recent published model-based estimates, separate categories for cancer risk factors are defined below based on their biologic nature, modifiability, and use in the references: ${ }^{1}$ Unmodifiable intrinsic risk, or mentioned as an intrinsic risk in this review, refers to unavoidable spontaneous mutations, which arise as a result of random errors in DNA replication; Non-intrinsic risk refers to exogenous and endogenous factors. They include modifiable exogenous factors e.g., carcinogens, xenobiotic, viruses, and lifestyle factors e.g., smoking, nutrient intake, physical activity, hormone therapy. The risks also include endogenous factors that are partially modifiable and related to the nature of an individual (e.g., hormone levels, immune response, DNA damage response, metabolism) and affect main aspects of cell growth control and genome integrity. ${ }^{10-12}$

\section{Intrinsic risk factors}

Unmodifiable intrinsic risk arises from the basal mutation rate occurring in all dividing cells despite the absence of any non-intrinsic factors. This risk corresponds to any biological intrinsic factor that causes unmodifiable DNA mutations. ${ }^{1}$ All humans have this kind of risk, which may vary among individuals. Driver mutations are required for cancer development."Passenger mutations"do not impact cancer formation but are commonly found in cancers. Passage or randomly acquired mutations in a tissue (e.g. deletions, single nucleotide errors, and insertions) depends on its progeny and also the survival and division of the mutated cell. However, it requires more than one driver mutation to initiate cancer increases the protection to develop cancer with only intrinsic mechanisms. Several studies have been undertaken to estimate the number of driver mutations required to develop an invasive carcinoma. The emerging consensus stated at least 3 hits are required for solid tumors and fewer for hematologic malignancies. ${ }^{1}$

The high rate of cancers in high incidence regions is most likely due to non-intrinsic factors, considering that unmodifiable intrinsic risk aroused from endogenous mutations should not show large geographic or time-dependent variations. The estimated percentages of lifetime risk of breast, colorectal, lung, prostate, and all cancers due to non-intrinsic factors in US (US_average-minimum)/US_average, are 93\%, 97\%, $99 \%, 99 \%$, and $88 \%$, respectively. The same percentages hold for UK. ${ }^{13}$

Table 1. Types of cancer risk factors ${ }^{1}$

\begin{tabular}{lll}
\hline Intrinsic Risk Factors & \multicolumn{2}{c}{ Non-Intrinsic Risk Factors } \\
\hline \multirow{2}{*}{ Unmodifiable } & Endogenous risk factors & Exogenous risk factors \\
& Partially modifiable & Modifiable \\
\cline { 2 - 3 } A random error of DNA & Biologic aging & Radiation \\
replication & Genetic susceptibility & Chemical carcinogens \\
& Epigenetics & Viral carcinogen \\
& Hormone & Unhealthy lifestyle (e.g. smoking, lack of \\
& Growth factors & physical activity, nutrition, etc \\
& Inflammation, etc & \\
\hline
\end{tabular}


Wu and Hannun have done recent studies on mutational signatures in cancer, which are regarded as residual evidence of cancer genomes in different mutagenic processes. ${ }^{14,4}$ Among approximately 30 distinct signatures, 2 were considered intrinsic processes since their strong positive correlations with age in the majority of cancers. The 2 signature mutations were presumed to result from extrinsic factors due to a lack of correlation with age. They found that the majority of cancers had a large proportion of mutations possibly caused by extrinsic factors. A few types of cancer had $>50 \%$ intrinsic mutations.

\section{Non-intrinsic risk factors}

There are multifaceted mechanisms of non-intrinsic risk factors, which are presumed to drive cancers. Some due to the family of chemicals that induce new mutations (mutagens), while other factors, such as viruses, induce cancers by activating oncogenes or inhibiting tumor suppressor genes in addition to inducing mutations. These mutagens operate on cells that can divide and persist to facilitate tumor development. They are 'at risk' cell populations; hence, biologic studies have focused on stem cells, progenitor cells, and other dividing cells. 'Non-intrinsic factors' was proposed to refer to risk factors other than intrinsic replication error, and includes not only exogenous factors (e.g., tobacco, ultraviolet (UV) radiation, drugs, and HPV) but also endogenous factors, such as inflammation, hormones, and growth factors, metabolic effects, reactive oxygen species, immune responses, etc. ${ }^{1,2}$

\section{Endogenous risk factors}

Hormones. Certain cancer risk factors are endogenous to the individual and many have some genetic component. One of the best examples among endogenous cancer risk factors studies is the individual levels of the sex steroid hormones and their role in the risk of breast cancer. As endogenous determinants of cancer risk, the steroid sex hormones levels vary over the life course and between individuals. The hormones also are influenced by other exogenous factors (e.g., diet, other drugs, therapeutic hormones, physical activity levels) and other endogenous determinants such as genetic factors. ${ }^{10-12}$

Obesity. Obesity-associated changes in metabolism, hormones, and inflammation are the biological culprits in cancer risk. Obesity has a genetic basis but most frequently develops from interaction with exogenous factors such as consumption of food, which are highly modifiable. For instance, the significantly increased risk of uterine cancer in obese females is closely related to deregulated sex hormones. ${ }^{10-12}$

Inflammation. Numerous cellular and molecular mechanism have been characterized to correlate inflammation and malignant cell persistence and invasion. These range from inflammation-induced Reactive Oxygen Species (ROS) that act in DNA damage to tumor initiation, as well as inflammation-derived cytokine and chemokine effects on tumor growth, angiogenesis, and tumor cell migration and invasion. Inflammation is accepted to act across the continuum of tumorigenesis in several cancer types. Such effects have led to the inclusion of inflammation as an enabling factor to carcinogenesis. ${ }^{15-18}$

Aging. Aging is considered among the most significant risk factors for cancer. It can be chronological or biological aging that contributes towards cancer development. The chronological aging role is allowing time for intrinsic risk as well as for exogenous and endogenous factors to take effect, while biological aging processes are more difficult to define or quantify since their full spectrum is not well defined yet. Some researchers have observed the impact of aging and revealed that the most abundant target of DNA chemical modification in mammalian cells is $\mathrm{CPG}$ dinucleotides. Age-associated changes to the mammalian DNA methylome are thought to promote diseases such as cancer. ${ }^{19}$ Podolski et al. reported a monotonic acceleration of age-associated CT and GA mutations at CpG sites. ${ }^{2}$ The mutation acceleration is higher in males and is earlier initiated in life in males. Future studies are required to determined tissue-specific, biological and chronological clocks that may be related to the dysfunctions of these functions of interests. ${ }^{19}$ Not all biological aging is pro-tumorigenic. ${ }^{20}$

\section{Tumor epigenetics}

Replication errors including epigenetic changes (e.g., DNA methylation) are passed on to daughter cells. Thus, our ability to comprehend and to design cancer etiology and the impacts of exogenous and endogenous risk factors should be extended to the area of many factors on the epigenome. There is convincing evidence that epigenetic changes not only occur during tumor development, but they also play a direct causal role e.g., specific epigenetic silencing of MLH-1 in a subset of human colon cancers, which are essential alterations in human 
tumorigenesis. Exogenous and endogenous factors including inflammation, obesity, and aging may similarly alter the thresholds and/or confer transformation via effects on the epigenome. ${ }^{20,21}$

\section{Exogenous risk factors \\ Infectious agents}

An interesting fact comes up, persistent virus infections are estimated to cause $20 \%$ of cancers worldwide varying from a few percent in the U.S. to $80 \%$ in some African countries in with AIDS as a cofactor. In the U.S., the major cancer-causing viruses are Human Papillomaviruses (HPVs), which cause cancers of the cervix, oropharynx, and several other sites. Hepatitis viruses cause liver cancer. Helicobacter pylori were identified as a potential causative agent $>30$ years ago, shown to increase the risk of gastric cancer.

HPV: Some of these strains are low risk and are associated with nonlethal outcomes, including genital warts, benign lesions of the oral cavity, and polyps. However, persistent infection with a subset of certain strains poses a high risk for cervical, anogenital, and oropharyngeal cancers and is associated with $\sim 5 \%$ of total cancers. Among the high-risk strains, HPV16 and HPV18 were the first recognized in studies of cervical cancers, while some other strains (e.g., HPV31 and HPV45) are also associated with high risk. A large study involving almost 5000 females in 2005-2006 disclosed that $\sim 40 \%$ of the participants were seropositive for any one of nine HPV strains evaluated, $21 \%$ of them are seropositive for the high-risk HPV16 and HPV18 strains. ${ }^{22}$

Hepatitis viruses: Uncontrolled infection with hepatitis viruses, including Hepatitis B Virus (HBV), Hepatitis C Virus (HCV), and Hepatitis D Virus (HDV), is a primary risk factor for Hepatocellular Carcinoma (HCC). These viruses pose a growing risk in some areas of the U.S., in part due to the growing population of immigrants from the east and south-east Asia where HBV infections are endemic. ${ }^{23,24}$

Helicobacter pylori: Helicobacter pylori colonization in the gut is one of the strong risk factors for gastritis, gastric ulcers, and stomach cancer although it is frequently asymptomatic at first. H.pylori is a Gram-negative bacillus. The cancer-promoting activity of H.pylori is expressed by bacterial proteins that induce a pathogenic inflammatory response; of these, the cytotoxin-associated A (cagA) gene has been most studied, and cagA+ strains of $H$. pylori are much more cancer-promoting than cagA-strains based on roles of cagA in reprogramming cancer-associated processes, including cell cycle, cell motility, epithelial-mesenchymal transition (EMT), and others. ${ }^{25-27}$

\section{HEREDITARY CANCER RISK ASSESSMENT}

Cancer susceptibility genes in individuals are now a common target of genetic testing in clinical practice to predict the risk of developing cancer. In general, to determine whether an individual carries a change that is likely to disrupt normal gene function, sequence-based testing of DNA is applied.

\section{Classification of cancer susceptibility genes}

Cancer susceptibility genes are classified into three main groups as follows: Genes in which mutations contribute to a high risk developing of cancer, although such mutations are rare (highly penetrant genes); Uncommon mutations in genes conferring a moderate increase of risk (odds ratios of approximately 2 - 4) (moderate penetrance genes); Common polymorphic variants (SNP's), which confer only slight risk alterations (odds ratios rarely above 1.2 for variants conferring increased risk) (low penetrance genes). These genes and loci were discovered on linkage analysis (for high-risk genes), screening for mutations in selected candidate genes because they were involved in functional pathways related to gene function (moderate risk), and genome-wide association studies (low penetrance polymorphisms). ${ }^{27,28}$

\section{Highly penetrant genes}

BRCA1 and BRCA2 mutations are considered as rare highly penetrant breast cancer-predisposing genes and account for $16-25 \%$ of the inherited component of breast cancer. Mutations in TP53, which provoke Li-Fraumeni syndrome, STK11 delivering Peutz-Jeghers syndrome, and PTEN causing Cowden syndrome, as are mutations in $\mathrm{CDH} 1$ are an infrequent causal agent of breast cancer, although they are considered as highly penetrant genes for breast cancer. ${ }^{29}$

\section{The intermediate penetrance cancer susceptibility genes}

Scientists have performed research on genes in which inherited mutations conferred an intermediate risk of cancer development. Interestingly, that mutations in ATM, CHEK2, BRIP1, BARD1, and PALB2 can cause an increased odds ratio 
for breast cancer of 2 - 4 because these genes are all involved in the same DNA repair pathways as BRCA although they do not confer the high risk of breast and ovarian cancer found in females who carry mutations in BRCA1 and BRCA2. It was found that Fanconi anemia subtypes FANC D, J, and N respectively were caused by biallelic mutations in BRCA2, BRIP1, and PALB2, implicating an overlapping function of these genes. Another gene, which interacts with BRCA1 and was involved in DNA repair is RSD50. These mutations are rare in the population and screening for such mutations would not be cost-effective unless there was a founder mutation conferring a significantly increased risk. ${ }^{28}$

\section{The common low-penetrance cancer susceptibility alleles}

The rapid increase in studies utilizing Single Nucleotide Polymorphisms (SNPs) has led to an abundance of references on this subject. The studies demonstrated that there was contradictory evidence of the importance of the role of such polymorphisms. Today, it has been established that there are up to eight polymorphisms that are reproducibly discovered to influence breast cancer risk, particularly the FGFR2 gene. Carriers of two low-risk rs2981582 alleles at the FGFR2 locus (38\% of the population) have a relative risk of breast cancer of 0.83 compared with the general population. Carriers who pose one high-risk and one low-risk allele (47\%) have a relative risk of 1.05 , and carriers of two high-risk alleles (14\%) have a relative risk of 1.26 . The overall risk of these polymorphisms remains high, an individual possessing several polymorphisms will have an increased risk of cancer. ${ }^{29}$

Figure 1 illustrates that very rare high-risk alleles (variants) are hard to find but may show a strong family aggregation, while low-risk alleles (variants) are commonly found in a population but do not show family aggregation and may be found in sporadic cancer. Although common variants are low-risk alleles, if there are several variants are found together in one individual, the risk for cancer in this individual may increase. Therefore, it is important to assess the risk of cancer through the use of multigene panels.

Throughout their lifetimes, all humans accumulate novel Pathogenic Variants (PVs), as a result of environmental exposures and the process of natural aging. Acquired Somatic Mosaicism (ASM) is a not uncommon laboratory finding. Pathogenic variants associated with ASM typically occur at extremely low allelic frequencies, which are not detectable by routine NGS analysis. However, some acquired PVs may deliver a selective growth advantage in hematopoietic cells conferring significant amplification due to clonal hematopoiesis. New generation sequencing analysis of blood samples may detect these amplified variants. This is particularly true for PVs in TP53, CHEK2, and ATM. ${ }^{8}$

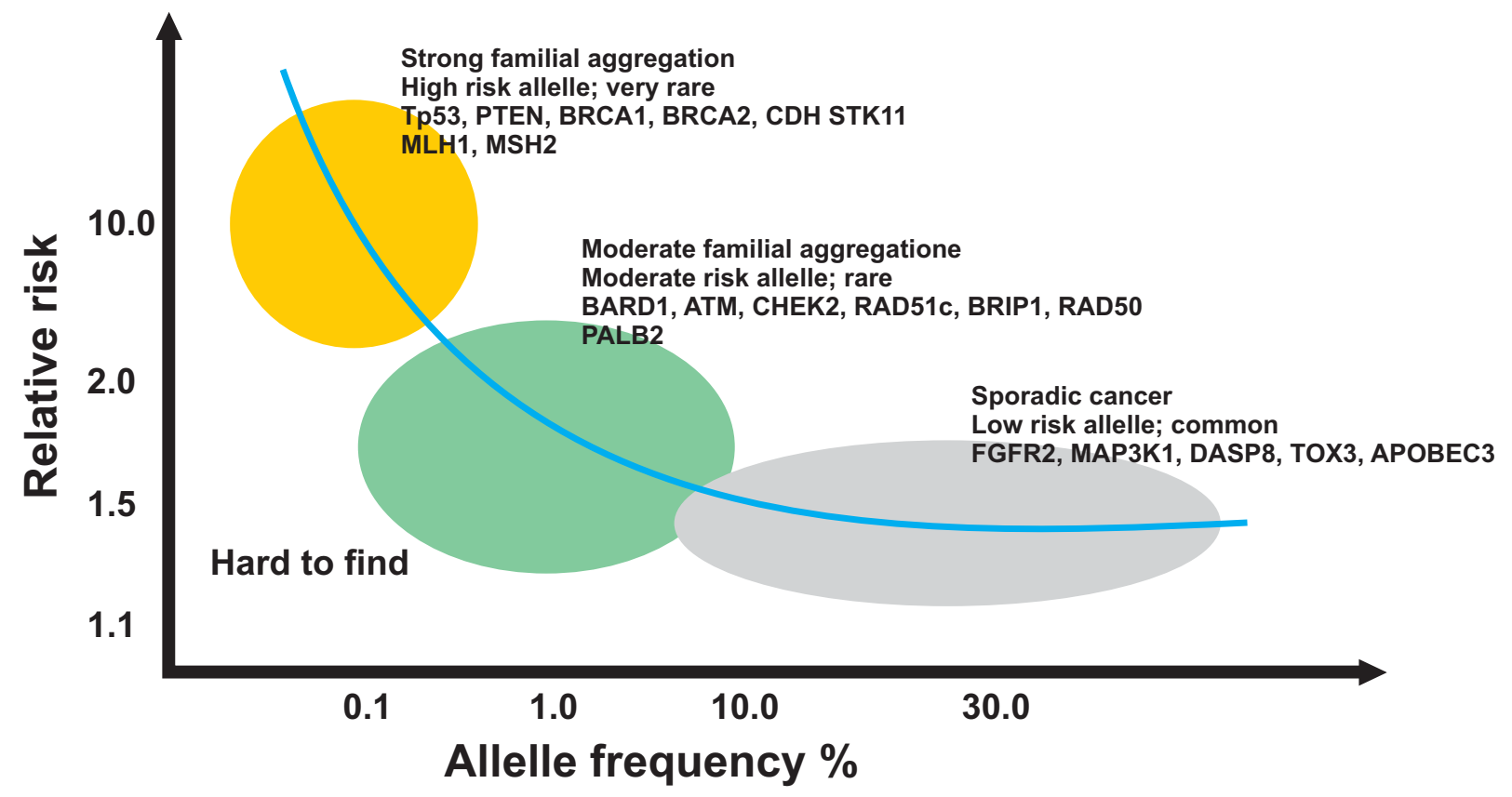

Figure 1. Relationship between relative risk and allele frequency (modified from O'Mara and Klonowska) 30,31 $^{30}$ 
Table 2. A classification system for sequence variants identified by genetic testing

\begin{tabular}{clc}
\hline Class & \multicolumn{1}{c}{ Description } & Likelihood of Pathogenicity \\
\hline 5 & Definitely pathogenic & $>0.99$ \\
4 & Likely pathogenic & $0.95-0.99$ \\
3 & Uncertain & $0.05-0.949$ \\
2 & Likely not pathogenic or of little clinical significance & $0.001-0.049$ \\
1 & Not pathogenic or of no clinical significance & $<0.001$ \\
\hline
\end{tabular}

\section{Classification of DNA variants}

Not all of the DNA variants are going to confer a disease or inherited. Plon et al. recommended five classes of a classification system that convey clearer information about the relevance of a variant to patient outcome including cancer risk assessment and the need for further studies. ${ }^{32,7}$ This classification avoids the use of the currently used term of unclassified (variants of unclassified/undetermined significance, VUS) in describing variants. (Table 2). Class 1 refers to the qualitative classification " Not Pathogenic" or "No Clinical Significance". It means any variant assigned to class 1 will not confer any major clinically significant effect on cancer risk. Class 2 corresponds to "likely not pathogenic" or of "Little Clinical Significance" with a likelihood of pathogenicity of $5 \%$. Class 3 ("Uncertain") is meant to differentiate variants with too little information to make any recommendation from variants with significant evidence against class 2 or for class 4 pathogenicity. Class 4 corresponds to "Likely Pathogenic" with a likelihood of pathogenicity of 95\%. The variant classification must be included within the main body of the clinical diagnostic report together with the gene-disease association and the mode of inheritance. ${ }^{29}$

\section{METHODS USED IN CANCER RISK ASSESSMENT}

The latest technologies currently used in cancer risk assessment for instance are quantitative PCR, copy number micro-array, Sanger sequencing, and NGS. $^{33}$ Commonly used technologies vary significantly in sensitivity, specificity, ease-of-use, and costs associated with the development and patient testing. Hereditary cancer testing typically is performed using gene panels, which may be either cancer-specific or for pan-cancer, to assess risk for a defined or broader range of cancers, respectively. Considering the clinical implications of gene testing, diagnostic laboratories must develop high-quality panel tests, which serve a broad and genetically diverse patient population. ${ }^{4.8}$

The ability of the assay to accurately identify variants and the knowledge of the pathologist to assign the correct clinical interpretation such as pathogenic or benign to each variant (i.e. interpretive accuracy) will determine overall test quality and accuracy. Failure to detect a Pathogenic Variant (PV) or erroneously reporting a PV as benign will lead to a false-negative result. On the other hand, a false-positive result will be obtained if the laboratory reports a PV that is not present in the patient or classifies a benign variant as pathogenic. Thus, accuracy requires a rigorous variant classification program, as well as robust lab-assays capable of detecting a wide variety of variants. Analytical accuracy should be optimized during assay development, validated to determine performance at implementation, and continuously monitored to ensure ongoing quality. ${ }^{9}$

Multi-gene panel NGS testing for hereditary Breast Cancer (BC) risk assessment is gaining acceptance and has proven to be useful as a diagnostic tool for disorders associated with specific phenotypes that can be influenced by multiple genes. However, NGS still has limitations. Nowadays, among females with an apparent predisposition to $B C$, multi-gene panel testing is preferred rather than single and two-gene tests. Multi-gene panels testing doubles the ability to detect pathogenic mutations related to cancer pathogenesis and increases the analysis of 6 to more than 100 genes simultaneously.

\section{CONCLUSION}

Multiple studies have shown that multi-gene testing identifies both expected and unexpected mutations and sometimes the genotype does not match the phenotype. Therefore, it is a challenge for the healthcare providers particularly the clinical pathologists who have to interpret the results, which implies the patient medical management. ${ }^{4}$ The benefits of genetic testing come from placing the 
results in the right clinical context to make the proper recommendations for diagnosis and management.

\section{REFERENCE}

1. Wu S, Zhu W, Thompson P, Hannun YA. Evaluating intrinsic and non-intrinsic cancer risk factors. Nature Communications, 2018; 9: 3490.

2. Podolski DI, Gladyshev VN. Intrinsic vs. extrinsic cancer risk factors and aging. Trends Mol Med, 2016; 22(10): 833-34.

3. Revelo AE, Martin A, Velasquez R, Kulandaisamy PC, Bustamante J, et al. Liquid biopsy for lung cancers: An update on recent developments. Ann Transl Med, 2019; 7(15): 349.

4. Hiraki S, Rinella ES, Schnabel F, Oratz R, Ostrer H. Cancer risk assessment using genetic panel testing: Consideration for clinical application. J Genet Counsel, 2014; 23: 604-617.

5. Al-Bader SB, Alsulaiman R, Bugrein H, Ben Omran $T$, Abbaszadeh $F$, et al. Cancer genetics program: Follow-up on clinical genetics and genomic medicine in Qatar. Molecular Genetics \& Genomic Medicine, 2018; 6(6):865-872.

6. Pruthi $S$, Heisey RE, Bevers TB. Chemoprevention for breast cancer. Ann Surg Oncol, 2015; 22(10): 3230-3235.

7. Turnbull C, Hodson S. Genetic predisposition to cancer. Clin Med, 2005; 5(5): 491-98.

8. Bowles KR, Mancini-DiNardo D, Coffee B, Cox HC, Qian $Y$, et al. Hereditary cancer testing challenges: Assembling the analytical pieces to solve the patients clinical puzzle. Future Oncol, 2019; 15(1): 65-79.

9. Horakova D, Bouchalova K, Cwiertka K. Risks and protective factors for triple negative breast cancer with a focus on micronutrients and infections. Biomed Pap Med Fac Univ Palacky Olomouc Czech Repub, 2018; 162(2): 83-89.

10. Doerstling SS, O'Flanagan $\mathrm{CH}$, Hursting SD. Obesity and cancer metabolism: A perspective on interacting tumor-intrinsic and extrinsic factors. Front Oncol, 2017; 7: 216.

11. Heymsfield SB, Wadden TA. Mechanisms, pathophysiology and management of obesity. $\mathrm{N}$ Engl J Med, 2017; 376(3): 254-66.

12. Vernieri C, Casola S, Folani M. Targeting cancer metabolism: Dietary and pharmacologic interventions. Cancer Discov, 2016; 6(12): 1315-33.

13. Zhu W, Wu S, Hannun YA. Contributions of the intrinsic mutation process to cancer mutation and risk burdens. E-Bio Medicine, 2017; 24: 5-6.

14. Wu S, Hannun Y. The importance of extrinsic factors in the development of cancers. Mol \& Cellular Oncol, 2016; 3(3): e1143079.

15. Elinav E. Inflammation-induced cancer: Crosstalks between tumors, immune cells and microorganisms. Nat Rev Cancer, 2013; 13: 759-71.

16. Mantovani A, Allavena P, Sica A. Cancer-related inflammation. Nature, 2008: 454:436-44.

17. Yu H, Pardoll D, Jove R. STAT's in cancer inflammation and immunity: A leading role for STAT3. Nat Rev Cancer, 2009; 9: 798-809.

18. Parker $\mathrm{KH}$, Beury DW, Ostrand-Rosenberg $\mathrm{S}$. Myeloid-derived suppressor cells: Critical Cells driving immune suppression in the tumor microenvironment. Advance in Cancer Research, 2015; 128: 95-139.

19. de Malgahaes, JP. How aging process influence cancer. Nat Rev Canc, 2013; 13: 357-65.

20. Donehover LA. MLH-1 silenced and non-silenced subgroups of hypermutated colorectal carcinomas have distinct mutational landscape. J Pathol, 2013; 229: 99-110.

21. Guida F. Dynamics of smoking-induced genome-wide methylation changes with time since smoking cessation. Hum Mol Genet, 2015; 24: 2349-59.

22. Schiller JT, Lowy DR. Understanding and learning from the success of prophylactic human papillomavirus vaccines. Nat Rev Microbiol, 2012; 10: 681-92.

23. Fujimoto $A$, Furuta $M$, Totoki $Y$. Whole genome mutational landscape and characterization of noncoding and structural mutations in liver cancer. Nat Genet, 2016; 48: 500-509.

24. McGlynn KA, London WT. The global epidemiology of hepatocellular carcinoma present and future. Clin Liver Dis, 2011; 15: 224-43.

25. Backert $S$, Blaser MJ. The role of CagA in the gastric biology of Helicobacter pylori. Cancer Res, 2016; 76: 4028-31.

26. Graham DY, Dore MP. Helicobacter pylori therapy: A paradigm shift. Expert Rev Anti Infect Therapy, 2016; 14:577-85.

27. Beggs AD, Hodgson SV, Genomics and breast cancer: The different levels of inherited susceptibility. Eur J Hum Gen, 2009; 17: 855-56.

28. Hodgson S. Mechanisms of inherited cancer susceptibility. J Zhejiang Univ Sc B. 2008; 9(1): 1-4.

29. Kobayashi H, Ohno S, Sasaki Y, Matsuura M. Hereditary breast and ovarian cancer susceptibility genes (review). Oncol Rep, 2013; 30: 1019-29.

30. O'Mara TA, Glubb DM, Kho PF, Thomson DJ, Spurdle AB. Genome-wide association studies of endometrial cancer: Latest developments and future direction. Cancer Epidemiol Biomarkers Prev, 2019; 28(7): 1095-1102.

31. Klonowska K, Ratatjska M, Wojciechowska $M$, Kozlowska P. Genetic predisposition to breast and/or ovarium cancer-focus on the candidate BARD-1 gene. Journal of Biotechnology, Computational Biology and Bionanotechnology, 2014; 95(3)C: 203-214 C.

32. Plon SE, Eccles DM, Easton D, Foulkes WD, Genuardi M, et al. For the IARC unclassified genetic variants working group. Sequence variant classification and reporting: Recommendations for improving the interpretation of cancer susceptibility genetic test reporting: recommendations for improving the interpretation of cancer susceptibility genetic test results. Hum Mutation, 2008; 29(11): 1282-1291.

33. Catana A, Apostu AP, Antemie RG. Multi-gene panel testing for hereditary breast cancer: Is it ready to be used?. Med \& Pharm Reports, 2019; 92(3): 220-225. 